\title{
Bulk nature of layered perovskite iridates beyond the Mott scenario: An approach from a bulk-sensitive photoemission study
}

\author{
A. Yamasaki, ${ }^{1}$ S. Tachibana, ${ }^{1}$ H. Fujiwara, ${ }^{2}$ A. Higashiya, ${ }^{3,4}$ A. Irizawa, ${ }^{5}$ O. Kirilmaz, ${ }^{6}$ F. Pfaff, ${ }^{6}$ P. Scheiderer, ${ }^{6}$ J. Gabel, ${ }^{6}$ \\ M. Sing, ${ }^{6}$ T. Muro, ${ }^{7}$ M. Yabashi, ${ }^{4}$ K. Tamasaku, ${ }^{4}$ H. Sato,${ }^{8}$ H. Namatame, ${ }^{8}$ M. Taniguchi,${ }^{8,9}$ A. Hloskovskyy, ${ }^{10}$ H. Yoshida, ${ }^{11}$ \\ H. Okabe, ${ }^{11}$ M. Isobe, ${ }^{11}$ J. Akimitsu, ${ }^{12}$ W. Drube, ${ }^{10}$ R. Claessen, ${ }^{6}$ T. Ishikawa, ${ }^{4}$ S. Imada, ${ }^{13}$ A. Sekiyama, ${ }^{2,4}$ and S. Suga ${ }^{2,4,5}$ \\ ${ }^{1}$ Faculty of Science and Engineering, Konan University, Kobe 658-8501, Japan \\ ${ }^{2}$ Graduate School of Engineering Science, Osaka University, Toyonaka, Osaka 560-8531, Japan \\ ${ }^{3}$ Faculty of Science and Engineering, Setsunan University, Neyagawa, Osaka 572-8508, Japan \\ ${ }^{4}$ RIKEN SPring-8 Center, Sayo, Hyogo 679-5148, Japan \\ ${ }^{5}$ Institute of Scientific and Industrial Research, Osaka University, Ibaraki, Osaka 567-0047, Japan \\ ${ }^{6}$ Physikalisches Institut, Universität Würzburg, D-97074 Würzburg, Germany \\ ${ }^{7}$ Japan Synchrotron Research Institute, Sayo, Hyogo 679-5198, Japan \\ ${ }^{8}$ Hiroshima Synchrotron Radiation Center, Hiroshima University, Higashi-Hiroshima, Hiroshima 739-0046, Japan \\ ${ }^{9}$ Graduate School of Science, Hiroshima University, Higashi-Hiroshima, Hiroshima 739-8526, Japan \\ ${ }^{10}$ DESY Photon Science, Deutsches Elektronen-Synchrotron, D-22603 Hamburg, Germany \\ ${ }^{11}$ National Institute for Materials Science, Tsukuba, Ibaraki 305-0044, Japan \\ ${ }^{12}$ College of Science and Engineering, Aoyama Gakuin University, Sagamihara, Kanagawa 252-5258, Japan \\ ${ }^{13}$ College of Science and Engineering, Ritsumeikan University, Kusatsu, Shiga 525-8577, Japan \\ (Received 27 October 2013; revised manuscript received 2 March 2014; published 24 March 2014)
}

\begin{abstract}
We present genuine bulk Ir $5 d j_{\text {eff }}$ states of layered perovskite iridates obtained by hard-x-ray photoemission spectroscopy (HAXPES) with $s$ - and $p$-polarized lights. HAXPES spectra of $\mathrm{Sr}_{2} \mathrm{IrO}_{4}$ and $\mathrm{Ba}_{2} \mathrm{IrO}_{4}$ are well reproduced by the quasiparticle density of states calculated by the local density approximation with dynamical mean-field theory (LDA + DMFT). It is demonstrated that the insulating nature of the iridates is triggered by antiferromagnetic correlation (Slater type) combined with electron correlation (Mott type). The extremely-lowenergy bulk-sensitive photoemission spectroscopy reveals "bad metallic" states in the paramagnetic phase of the iridates, suggesting strongly renormalized metallic states above the Néel temperature as predicted by the LDA + DMFT.
\end{abstract}

DOI: 10.1103/PhysRevB.89.121111

Mott physics, which explains the insulating nature in materials triggered by strong electron correlations, has been vigorously studied for several decades from both experimental and theoretical points of view [1]. Owing to these efforts, new mechanisms for the metal-insulator transition (MIT) were proposed in some "Mott" systems, for instance, orbital switching for $\mathrm{VO}_{2}$ and the essential role of antiferromagnetic (AF) correlation effects for $\mathrm{La}_{2} \mathrm{CuO}_{4}[2,3]$.

Layered perovskite iridates $A_{2} \mathrm{IrO}_{4}(A=\mathrm{Sr}, \mathrm{Ba})$ have an insulating state, the origin of which was mysterious in the early stage of the research [4]. Detailed spectroscopic and theoretical studies recently demonstrated $\mathrm{Sr}_{2} \mathrm{IrO}_{4}$ to be a spin-orbit (SO) -driven Mott insulator [5]. According to that scenario, the electronic structure near the Fermi level $\left(E_{F}\right)$ is characterized by Kramers-doublet Ir $5 d j_{\text {eff }}=1 / 2$ states, which are eigenstates of the SO Hamiltonian within the $t_{2 g}$ subspace. For the iridate with five $5 d$ electrons, the lower $j_{\text {eff }}=3 / 2$ bands are fully occupied and the higher half-filled $j_{\text {eff }}=1 / 2$ band split into the upper and lower Hubbard bands due to the relatively weak on-site Coulomb interaction $(U)$. This simple picture has been widely accepted since the results of experiments, such as optical conductivity spectra, seemed to be well explained $[5,6]$. In addition, the results of angle-resolved ultraviolet photoemission spectroscopy were well reproduced by the local density approximation (LDA) $+U+\mathrm{SO}$ band structure calculations [5]. For $5 d$ transition-metal systems, however, the nature of atom-band duality, which is a characteristic feature of heavy elements, makes the interpretation of optical
PACS number(s): 79.60.-i, 71.70.Ej, 71.20.-b

conductivity spectra complicated [7]. In addition, we point out here that the photoemission spectra excited by several tens-eV photons are strongly affected by the surface electronic structures modified by stronger electron correlation effect on the surface than in the bulk even for quasi-two-dimensional compounds [8,9].

Recently, the LDA study with dynamical mean-field theory (LDA + DMFT) pointed out that both $\mathrm{Sr}_{2} \mathrm{IrO}_{4}$ and $\mathrm{Ba}_{2} \mathrm{IrO}_{4}$ should be classified as Slater insulators, in which the AF correlation mainly contributed to realizing the insulating states in these materials rather than the electron correlation [10,11]. Then, the time-resolved optical experiment revealed in $\mathrm{Sr}_{2} \mathrm{IrO}_{4}$ two different characteristic behaviors, each of which could be explained by Slater or Mott physics [12].

In this Rapid Communication, we focus on the layered perovskite iridates $\mathrm{Sr}_{2} \mathrm{IrO}_{4}$ and $\mathrm{Ba}_{2} \mathrm{IrO}_{4}$ which have comparable Néel temperatures $T_{N}(\simeq 250$ and $240 \mathrm{~K}$ ) but different crystal symmetries $\left(I 4_{1} /\right.$ acd and $\left.I 4 / \mathrm{mmm}\right)[13,14]$. It is an intriguing problem whether both iridates have a common insulating nature or not. Detailed information on their bulk electronic structures facilitates discussions about not only Mott and Slater physics but also a possibility of superconductivity, attracting a wide general interest $[15,16]$. In order to provide much deeper insight into the nature of the SO-induced insulating states, we have carried out soft-x-ray and hard-x-ray photoemission spectroscopy (SXPES and HAXPES) in the photon energy $(h v)$ ranges of $400-1200 \mathrm{eV}$ and 6-8 keV, respectively. HAXPES reveals genuine bulk $j_{\text {eff }}$ states owing to the large 
photoionization cross section $(\sigma)$ of Ir $5 d$ states [17] and the long inelastic mean-free path $\left(\lambda_{\mathrm{mp}}\right)$ of detected photoelectrons $[18,19]$. These findings are compared to the results of the LDA + DMFT calculations [10]. Furthermore, the extremelylow-energy photoemission spectroscopy (ELEPES) has been performed to reveal the bulk electronic states in the vicinity of $E_{F}$. ELEPES demonstrates that both $\mathrm{Sr}_{2} \mathrm{IrO}_{4}$ and $\mathrm{Ba}_{2} \mathrm{IrO}_{4}$ have "bad metallic" states above $T_{N}$ as predicted by the calculations.

HAXPES was performed at the long-undulator beamline BL19LXU in SPring-8 [20] with the MB Scientific A-1 $\mathrm{HE}$ spectrometer for $\mathrm{Sr}_{2} \mathrm{IrO}_{4}$ and at the undulator beamline P09 in Petra III at DESY [21] with the SPECS PHOIBOS $225 \mathrm{HV}$ spectrometer for $\mathrm{Ba}_{2} \mathrm{IrO}_{4}$. SXPES was carried out at the twin-helical undulator beamline BL25SU in SPring-8 using the GAMMADATA-SCIENTA SES-200 spectrometer [22]. The undulators in the SX and HAX beamlines produce circularly and linearly polarized lights, respectively. ELEPES experiments were performed with the MB Scientific T- 1 xenon discharge lamp [23,24] and the A-1 spectrometer at Konan University. Inverse photoemission spectroscopy (IPES) was performed in HiSOR at Hiroshima University [25]. For all the measurements, polycrystalline samples were used [14]. Clean surfaces were obtained by fracturing samples in situ in $\mathrm{UHV}\left(\leqslant 2 \times 10^{-7} \mathrm{~Pa}\right.$ for HAXPES, $\leqslant 3 \times 10^{-8} \mathrm{~Pa}$ for SXPES, XAS, ELEPES, and IPES). The total energy resolution $(\Delta E)$ of each experiment was estimated by the Fermi edge of gold.

Figure 1(a) shows valence-band PES spectra of $\mathrm{Sr}_{2} \mathrm{IrO}_{4}$ at three different $h v$ 's. Both Ir $5 d$ and $\mathrm{O} 2 p$ states have large spectral weight in the SXPES spectrum measured at $h \nu=450 \mathrm{eV}$ since the $\sigma$ 's of these states are comparable $\left(\sigma_{\text {Ir } 5 d^{5}} / \sigma_{\left(\mathrm{O} 2 p^{6}\right)_{4}} \simeq 0.6\right)$ and larger than those of other states. In contrast, the HAXPES spectrum measured at $h v \simeq 8000 \mathrm{eV}$ originates mainly from the Ir $5 d$ states due to the much larger $\sigma$ than those of other states, for instance, $\sigma_{\mathrm{Ir} 5 d^{5}} / \sigma_{\left(\mathrm{O} 2 p^{6}\right)_{4}} \simeq 80$. Therefore, by comparing these spectra, one can conclude that a large peak at the binding energy $\left(E_{B}\right)$ of $3.4 \mathrm{eV}$ (denoted as $A$ ), and two small peaks located at $10.3 \mathrm{eV}(B)$ and $12.5 \mathrm{eV}(C)$ contain strong $\mathrm{O} 2 p$ components. They are derived from the $\mathrm{O}$ $2 p$ nonbonding states $(A)$ and $\operatorname{Ir} 5 d e_{g}-\mathrm{O} 2 p$ bonding states $(B$ and $C$ ), respectively. The HAXPES spectrum has a two-peak structure in which the lower- and higher- $E_{B}$ peaks (at $E_{B} \simeq 1$ and $6 \mathrm{eV}$ ) originate from the Ir $5 d j_{\text {eff }}-\mathrm{O} 2 p$ antibonding states and bonding states, respectively.

We note that the photoelectron emission utilizing linearly polarized light has angular distributions depending on the orbital symmetry of electrons. In the HAXPES experiments for $\mathrm{Sr}_{2} \mathrm{IrO}_{4}$ two different configurations with $p$ and $s$ polarizations (the degrees of the linear polarization $P_{L}$ 's are +0.98 and -0.8 [26], respectively) were employed as illustrated in Fig. 1(d) [27]. One can expect to suppress the spectral weight derived mainly from $s$ states in the spectrum measured with the $s$ polarized light. Meanwhile, the value of $\sigma_{\mathrm{Ir} 5 d^{5}} / \sigma_{\left(\mathrm{O} 2 p^{6}\right)_{4}}$ for the $s$-polarized light is smaller ( $\sim 20$ at $h v=8 \mathrm{keV}$ in BL19LXU) than that for the $p$-polarized light $(\sim 80$ at $h v=8 \mathrm{keV}$ in BL19LXU and $\sim 90$ at $h v=6 \mathrm{keV}$ in P09) [28]. As can be seen in Fig. 1(c), the reduction of the spectral weight associated with the polarization change $(p \rightarrow s)$ has never been observed in the $j_{\text {eff }}$ antibonding bands $\left(0 \leqslant E_{B} \leqslant \sim 3 \mathrm{eV}\right)$, clearly indicating
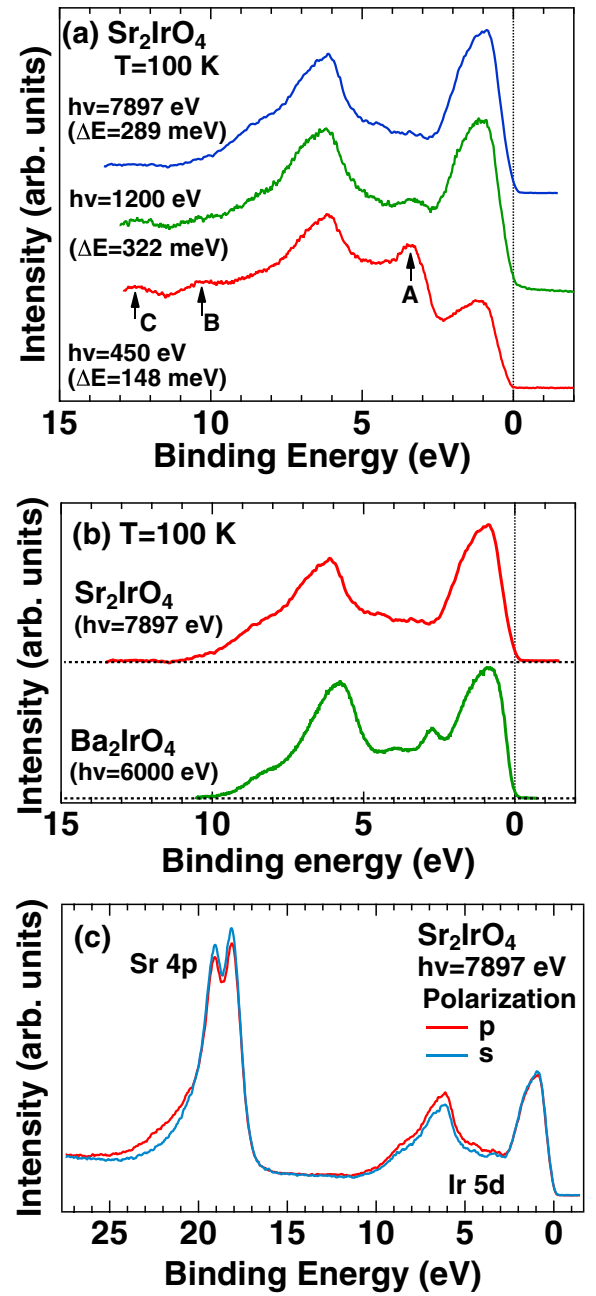

(d) $\mathrm{Sr}_{2} \mathrm{IrO}_{4}$

BL19LXU (top view)

$\mathrm{Ba}_{2} \mathrm{IrO}_{4}$
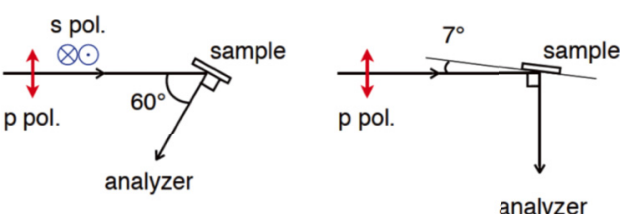

FIG. 1. (Color online) (a) Valence-band SXPES and HAXPES spectra of $\mathrm{Sr}_{2} \mathrm{IrO}_{4}$. (b) Valence-band HAXPES spectra of $\mathrm{Sr}_{2} \mathrm{IrO}_{4}$ and $\mathrm{Ba}_{2} \mathrm{IrO}_{4}$ after subtracting Shirley-type backgrounds. Both spectra were obtained for $p$-polarized lights. (c) Polarization-dependent HAXPES spectra of $\mathrm{Sr}_{2} \mathrm{IrO}_{4}$. (d) Schematic illustrations of experimental setups for HAXPES.

that the contributions of any $s$ states are negligibly small in this $E_{B}$ region. Hereafter, we discuss the bulk Ir $5 d j_{\text {eff }}$ electronic structures based on the results of the HAXPES with the $p$-polarized light because of the highest $\sigma$ ratio of $\operatorname{Ir} 5 d$ to O $2 p$ states.

Now one can directly compare the HAXPES spectra representing genuine bulk $j_{\text {eff }}$ states with the results of the LDA + DMFT calculations for iridates. Details of the calculation method have been reported elsewhere [10]. Figure 2(a) shows the HAXPES spectra in the $j_{\text {eff }}$ states. The LDA + DMFT spectra calculated for the antiferromagnetic insulating (AFI) 

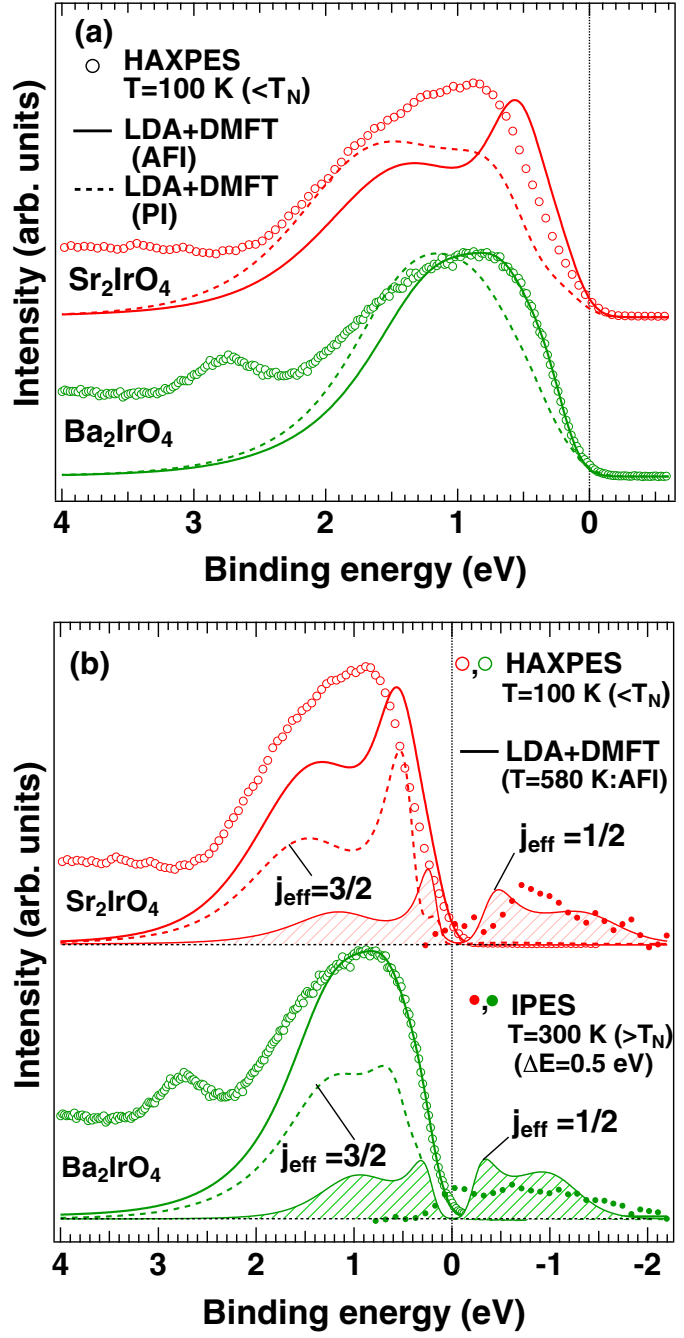

FIG. 2. (Color online) Valence-band HAXPES spectra in $j_{\text {eff }}$ states of $\mathrm{Sr}_{2} \mathrm{IrO}_{4}$ and $\mathrm{Ba}_{2} \mathrm{IrO}_{4}$. (a) Calculated spectra for both AFI (solid line) and PI (dashed line) phases are shown, which are broadened by Gaussian and Lorentzian functions representing the experimental energy-resolution and lifetime effects. Finite temperature effect around $E_{F}$ is also considered. (b) IPES spectra measured for the kinetic energy of incident electrons, $E_{K}=50 \mathrm{eV}$ at $T=300 \mathrm{~K}$ (above $T_{N}$ ) are added (indicated by solid circles). Calculated spectra of both $j_{\text {eff }}=3 / 2$ (dashed line) and $1 / 2$ (solid line with hatches) states for the AFI phase are also shown separately. All the experimental spectra are normalized by the area under the curves after subtracting Shirley-type backgrounds [see Fig. 1(b)].

ground states $\left(U=1.96 \mathrm{eV}\right.$ for $\mathrm{Sr}_{2} \mathrm{IrO}_{4}$ and $1.6 \mathrm{eV}$ for $\left.\mathrm{Ba}_{2} \mathrm{IrO}_{4}\right)$ and the paramagnetic insulating (PI) states $(U=$ $2.4 \mathrm{eV}$ for $\mathrm{Sr}_{2} \mathrm{IrO}_{4}$ and $1.8 \mathrm{eV}$ for $\mathrm{Ba}_{2} \mathrm{IrO}_{4}$ ) are also shown in Fig. 2(a). The calculated spectra of $\mathrm{Sr}_{2} \mathrm{IrO}_{4}$ and $\mathrm{Ba}_{2} \mathrm{IrO}_{4}$ for the PI phase (indicated by dotted lines, with larger $U$ values than the critical value $U_{c}$ for Mott transition) cannot reproduce the experimental spectra at all in the vicinity of $E_{F}$. Therefore, the insulating nature of both iridates can never be described by the simple Mott picture. Meanwhile, the calculated spectra for the AFI phase of $\mathrm{Sr}_{2} \mathrm{IrO}_{4}$ and $\mathrm{Ba}_{2} \mathrm{IrO}_{4}$ qualitatively reproduce the experimental spectra, supporting the "Slater-dominant" transition scenario for these compounds [10,29].
We note that the DMFT study strongly overestimated their $T_{N}$ 's $\left(810 \mathrm{~K}\right.$ for $\mathrm{Sr}_{2} \mathrm{IrO}_{4}$ and $690 \mathrm{~K}$ for $\left.\mathrm{Ba}_{2} \mathrm{IrO}_{4}\right)$ without thought of both intersite fluctuations and interactions between crystal layers. While the HAXPES spectra were measured at $T=100 \mathrm{~K}\left(140-150 \mathrm{~K}\right.$ below the real $T_{N}$ and this difference is defined as $\left.\Delta T_{\mathrm{PES}}\right)$, the LDA + DMFT spectra of $\mathrm{Sr}_{2} \mathrm{IrO}_{4}$ and $\mathrm{Ba}_{2} \mathrm{IrO}_{4}$ in Fig. 2(a) were calculated for $T=580 \mathrm{~K}(230$ and $110 \mathrm{~K}$ below the calculated $T_{N}$ 's, respectively, and these differences are defined as $\left.\Delta T_{\mathrm{DMFT}}\right)$. The larger discrepancy between $\Delta T_{\mathrm{PES}}$ and $\Delta T_{\mathrm{DMFT}}$ in $\mathrm{Sr}_{2} \mathrm{IrO}_{4}$ yields less agreement between the experimental and theoretical spectra than in $\mathrm{Ba}_{2} \mathrm{IrO}_{4}$ in the empirical point of view.

The Slater picture provides a metallic nature above $T_{N}$ unlike the Mott picture. In general, the gap opens far above $T_{N}$ in Mott systems [30]. In order to investigate the spectral behavior near $E_{F}$, the ELEPES spectra have been measured at $T=300 \mathrm{~K}$ for $\mathrm{Sr}_{2} \mathrm{IrO}_{4}$ and $\mathrm{Ba}_{2} \mathrm{IrO}_{4}$. According to the "universal" curve between $\lambda_{\text {mp }}$ in solids and the photoelectron kinetic energy [32], photoelectrons excited by Xe I $\alpha$ light are able to provide the information on the bulk far from the surface [33-35]. The bulk sensitivity in ELEPES is, however, known to depend on individual material systems, or in other words, on the dielectric function, band structures, and partial density of states (PDOS) near $E_{F}$ [36-38]. The low PDOS near $E_{F}$ is thought to realize the enhanced $\lambda_{\text {mp }}$ at $h v=8.4 \mathrm{eV}(\mathrm{Xe}$ $\mathrm{I} \alpha$ ) [38]. Figure 3(a) shows the ELEPES spectrum of $\mathrm{Sr}_{2} \mathrm{RhO}_{4}$
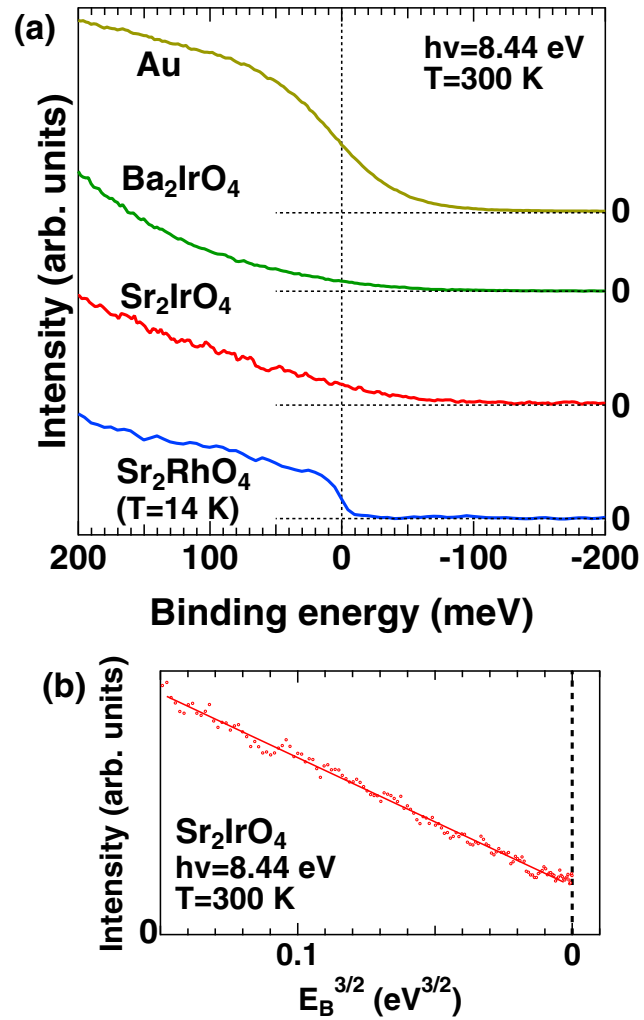

FIG. 3. (Color online) (a) ELEPES spectra of $\mathrm{Sr}_{2} \mathrm{IrO}_{4}$ and $\mathrm{Ba}_{2} \mathrm{IrO}_{4}$ near $E_{F}$ at $T=300 \mathrm{~K}$ (above $T_{N}$ ). The ELEPES spectrum of metallic $\mathrm{Sr}_{2} \mathrm{RhO}_{4}$ at $T=14 \mathrm{~K}$ is also shown as a reference. (b) The ELEPES spectrum of $\mathrm{Sr}_{2} \mathrm{IrO}_{4}$ divided by Femi-Dirac distribution function. The spectrum is plotted as a function of $(\text { binding energy) })^{3 / 2}+$ constant. 
which has a clear Fermi cutoff. This indicates that ELEPES has a longer probing depth than low- $h v$ SXPES, in which the Fermi cutoff is unclear, in this system and can reveal the bulk electronic structure. It would be reasonable that one can expect higher bulk sensitivity for $\mathrm{Sr}_{2} \mathrm{IrO}_{4}$ because of less conduction electrons [38].

Figure 3(a) shows the ELEPES spectra of $\mathrm{Sr}_{2} \mathrm{IrO}_{4}$ and $\mathrm{Ba}_{2} \mathrm{IrO}_{4}$ at $T=300 \mathrm{~K}$, that is, above $T_{N}$ [39]. Although the $\sigma$ of Ir $5 d$ states is much smaller than that of $\mathrm{O} 2 p$ states $\left(\sigma_{\text {Ir } 5 d^{5}} / \sigma_{\left(\mathrm{O} 2 p^{6}\right)_{4}} \simeq 0.06\right)$ at $h v=8.4 \mathrm{eV}$, the spectral weight near $E_{F}$ is visible. Significantly weak intensities at $E_{F}$ and no Fermi cutoff were observed unlike the gold, suggesting "bad metallic" states in the paramagnetic phase. This is consistent with the results of IPES at $T=300 \mathrm{~K}$, in which the spectra have finite intensities at $E_{F}$ [see Fig. 2(b)]. The spectra of both iridates have some spectral weight in the optical "Mott" gap of $200 \mathrm{meV}$ below $E_{F}[6,40]$. According to the LDA + DMFT results, the energy gap is closed above $T_{N}$ due to the strongly renormalized quasiparticle spectral weight at $E_{F}$.

The ELEPES spectra of $\mathrm{Sr}_{2} \mathrm{IrO}_{4}$ follow a $3 / 2$ power law in the range from $E_{F}$ to $E_{B} \simeq 0.3 \mathrm{eV}$ as shown in Fig. 3(b). The $3 / 2$ power-law behavior was also observed in other iridates, such as ferromagnetic $\mathrm{BaIrO}_{3}$ and weakferromagnetic $\mathrm{Y}_{2} \mathrm{Ir}_{2} \mathrm{O}_{7}$, which was explained as a result of a dominant contribution of ferromagnetic magnons to the electron excitation [41,42]. A recent resonant inelastic x-ray scattering study revealed AF linear dispersion of magnons in $\mathrm{Sr}_{2} \mathrm{IrO}_{4}$ [40], indicating that one could observe some features derived from the $\mathrm{AF}$ magnon excitation rather than the ferromagnetic one. In contrast, the spectra of $\mathrm{Ba}_{2} \mathrm{IrO}_{4}$ have never followed the power law (not shown here). It is inferred that the spectral shape in ELEPES is affected by the unoccupied density of states and matrix element effects as well as electron correlations in the final state unlike in the high-energy PES. Although the origin of the different behaviors between these iridates remains to be clarified, it is probably associated with the unoccupied $\mathrm{Sr} 4 d$ and $\mathrm{Ba}$ $5 d$ bands (at $\sim 8 \mathrm{eV}$ above $E_{F}$ ), crystal symmetries, and the rotation of $\mathrm{IrO}_{6}$ octahedrons.

In conclusion, the HAXPES experiments have revealed the bulk insulating nature of the layered perovskite iridates $\mathrm{Sr}_{2} \mathrm{IrO}_{4}$ and $\mathrm{Ba}_{2} \mathrm{IrO}_{4}$. The simple Mott picture cannot reproduce the overall valence-band spectral features. ELEPES demonstrated that both $\mathrm{Sr}_{2} \mathrm{IrO}_{4}$ and $\mathrm{Ba}_{2} \mathrm{IrO}_{4}$ have "bad metallic" states in the paramagnetic phase, suggesting strongly renormalized metallic states above the Néel temperature as predicted by the LDA + DMFT calculations.

We would like to thank T. Aso, Y. Nishitani, T. Mori, and Y. Matsui at Konan University; S. Kitayama and T. Matsumoto at Osaka University; and J. Kodama and H. Nagata at Hiroshima University for supporting experiments; R. Arita and M. Imada at University of Tokyo for providing the results of band structure calculations and fruitful discussions; and N. Tomita at Yamagata University for fruitful discussions. The soft-x-ray experiments at SPring- 8 and the IPES experiments at HiSOR were performed with the approval of the Japan Synchrotron Radiation Research Institute (JASRI) (Proposal No. 2010A1227) and the approval of Hiroshima Synchrotron Radiation Center (Proposal No. 12-B-29), respectively, under the support of Grant-in-Aid for Scientific Research for Young Scientists (B) (Grant No. 23740244) from the Ministry of Education, Culture, Sports, Science, and Technology, Japan, and Research Foundation for Research Institute of Konan University. Work in Würzburg is supported by DFG (FOR 1162). The HAXPES instrument at beamline P09 is jointly operated by the University of Würzburg (R. Claessen), the University of Mainz (C. Felser), and DESY. Funding by the Federal Ministry of Education and Research (BMBF) under Contracts No. 05KS7UM1, No. 05K10UMA, No. 05KS7WW3, and No. 05K10WW1 is gratefully acknowledged.
[1] M. Imada, A. Fujimori, and Y. Tokura, Rev. Mod. Phys. 70, 1039 (1998)

[2] M. W. Haverkort, Z. Hu, A. Tanaka, W. Reichelt, S. V. Streltsov, M. A. Korotin, V. I. Anisimov, H. H. Hsieh, H.-J. Lin, C. T. Chen, D. I. Khomskii, and L. H. Tjeng, Phys. Rev. Lett. 95, 196404 (2005).

[3] A. Comanac, L. de' Medici, M. Capone, and A. J. Mills, Nat. Phys. 4, 287 (2008).

[4] R. J. Cava, B. Batlogg, K. Kiyono, H. Takagi, J. J. Krajewski, W. F. Peck, Jr., L. W. Rupp, Jr., and C. H. Chen, Phys. Rev. B 49, 11890 (1994).

[5] B. J. Kim, H. Jin, S. J. Moon, J.-Y. Kim, B.-G. Park, C. S. Leem, J. Yu, T. W. Noh, C. Kim, S.-J. Oh, J.-H. Park, V. Durairaj, G. Cao, and E. Rotenberg, Phys. Rev. Lett. 101, 076402 (2008).

[6] S. J. Moon, H. Jin, W. S. Choi, J. S. Lee, S. S. A. Seo, J. Yu, G. Cao, T. W. Noh, and Y. S. Lee, Phys. Rev. B 80, 195110 (2009).

[7] B. H. Kim, G. Khaliullin, and B. I. Min, Phys. Rev. Lett. 109, 167205 (2012).
[8] A. Sekiyama, T. Iwasaki, K. Matsuda, Y. Saitoh, Y. Ōnuki, and S. Suga, Nature (London) 403, 396 (2000).

[9] A. Sekiyama, S. Kasai, M. Tsunekawa, Y. Ishida, M. Sing, A. Irizawa, A. Yamasaki, S. Imada, T. Muro, Y. Saitoh, Y. Onuki, T. Kimura, Y. Tokura, and S. Suga, Phys. Rev. B 70, 060506(R) (2004).

[10] R. Arita, J. Kuneš, A. V. Kozhevnikov, A. G. Eguiluz, and M. Imada, Phys. Rev. Lett. 108, 086403 (2012).

[11] J. C. Slater, Phys. Rev. 82, 538 (1951).

[12] D. Hsieh, F. Mahmood, D. H. Torchinsky, G. Cao, and N. Gedik, Phys. Rev. B 86, 035128 (2012).

[13] M. K. Crawford, M. A. Subramanian, R. L. Harlow, J. A. Fernandez-Baca, Z. R. Wang, and D. C. Johnston, Phys. Rev. B 49, 9198 (1994).

[14] H. Okabe, M. Isobe, E. Takayama-Muromachi, A. Koda, S. Takeshita, M. Hiraishi, M. Miyazaki, R. Kadono, Y. Miyake, and J. Akimitsu, Phys. Rev. B 83, 155118 (2011).

[15] F. Wang and T. Senthil, Phys. Rev. Lett. 106, 136402 (2011).

[16] H. Watanabe, T. Shirakawa, and S. Yunoki, Phys. Rev. Lett. 110, 027002 (2013). 
[17] J. J. Yeh and I. Lindau, At. Data Nucl. Data Tables 32, 1 (1985).

[18] A. Yamasaki, S. Imada, H. Higashimichi, H. Fujiwara, T. Saita, T. Miyamachi, A. Sekiyama, H. Sugawara, D. Kikuchi, H. Sato, A. Higashiya, M. Yabashi, K. Tamasaku, D. Miwa, T. Ishikawa, and S. Suga, Phys. Rev. Lett. 98, 156402 (2007); A. Yamasaki, Y. Matsui, S. Imada, K. Takase, H. Azuma, T. Muro, Y. Kato, A. Higashiya, A. Sekiyama, S. Suga, M. Yabashi, K. Tamasaku, T. Ishikawa, K. Terashima, H. Kobori, A. Sugimura, N. Umeyama, H. Sato, Y. Hara, N. Miyagawa, and S. I. Ikeda, Phys. Rev. B 82, 184511 (2010).

[19] S. Tanuma, C. J. Powell, and D. R. Penn, Surf. Interface Anal. 21, 165 (1994).

[20] M. Yabashi, K. Tamasaku, and T. Ishikawa, Phys. Rev. Lett. 87, 140801 (2001).

[21] J. Strempfer, S. Francoual, D. Reuther, D. K. Shukla, A. Skaugen, H. Schulte-Schrepping, T. Kracht, and H. Franz, J. Synchrotron Radiat. 20, 541 (2013).

[22] Y. Saitoh, H. Kimura, Y. Suzuki, T. Nakatani, T. Matsushita, T. Muro, T. Miyahara, M. Fujisawa, K. Soda, S. Ueda, H. Harada, M. Kotsugi, A. Sekiyama, and S. Suga, Rev. Sci. Instrum. 71, 3254 (2000).

[23] S. Souma, T. Sato, T. Takahashi, and P. Baltzer, Rev. Sci. Instrum. 78, 123104 (2007).

[24] S. Suga, A. Sekiyama, G. Funabashi, J. Yamaguchi, M. Kimura, M. Tsujibayashi, T. Uyama, H. Sugiyama, Y. Tomida, G. Kuwahara, S. Kitayama, K. Fukushima, K. Kimura, T. Yokoi, K. Murakami, H. Fujiwara, Y. Saitoh, L. Plucinski, and C. M. Schneider, Rev. Sci. Instrum. 81, 105111 (2010).

[25] H. Sato, T. Kotsugi, S. Senba, H. Namatame, and M. Taniguchi, J. Synchrotron Radiat. 5, 772 (1998).

[26] $P_{L}$ is defined as $\left(I_{p}-I_{s}\right) /\left(I_{p}+I_{s}\right)$, where $I_{p}$ and $I_{s}$ stand for the intensities of $p$ - and $s$-polarization components in the x ray, respectively.

[27] A. Sekiyama, J. Yamaguchi, A. Higashiya, M. Obara, H. Sugiyama, M. Y. Kimura, S. Suga, S. Imada, I. A. Nekrasov, M. Yabashi, K. Tamasaku, and T. Ishikawa, New J. Phys. 12, 043045 (2010).

[28] M. B. Trzhaskovskaya, V. I. Nefedov, and V. G. Yarzhemsky, At. Data Nucl. Data Tables 77, 97 (2001); 82, 257 (2002); M. B. Trzhaskovskaya, V. K. Nukulin, V. I. Nefedov, and V. G. Yarzhemsky, ibid. 92, 245 (2006).

[29] The peak at $E_{B}=2.8 \mathrm{eV}$ in $\mathrm{Ba}_{2} \mathrm{IrO}_{4}$ originates from $\mathrm{O}$ $2 p$ nonbonding states and does not appear in the calculated spectrum.
[30] For instance, $\mathrm{V}_{2} \mathrm{O}_{3}$ is a typical Mott system. But the energy gap opens suddenly at $T_{N}$, since it is identical to the MIT temperature in this material. Across the $T_{N}$ the photoemission spectral shape drastically changes all over the valence band [31]. This is not the present case since we have never observed such sharp changes.

[31] H. Fujiwara, A. Sekiyama, S.-K. Mo, J. W. Allen, J. Yamaguchi, G. Funabashi, S. Imada, P. Metcalf, A. Higashiya, M. Yabashi, K. Tamasaku, T. Ishikawa, and S. Suga, Phys. Rev. B 84, 075117 (2011).

[32] D. A. Shirley, Photoemission in Solids I, edited by M. Cardona and L. Ley, Topics in Applied Physics Vol. 26 (Springer-Verlag, Berlin, 1978), p. 193.

[33] T. Kiss, F. Kanetaka, T. Yokoya, T. Shimojima, K. Kanai, S. Shin, Y. Onuki, T. Togashi, C. Zhang, C. T. Chen, and S. Watanabe, Phys. Rev. Lett. 94, 057001 (2005).

[34] J. D. Koralek, J. F. Douglas, N. C. Plumb, Z. Sun, A. V. Federov, M. M. Murnane, H. C. Kapteyn, S. T. Cundiff, Y. Aiura, K. Oka, H. Eisaki, and D. S. Dessau, Phys. Rev. Lett. 96, 017005 (2006).

[35] T. Sato, S. Souma, K. Sugawara, K. Nakayama, S. Raj, H. Hiraka, and T. Takahashi, Phys. Rev. B 76, 113102 (2007).

[36] D. R. Penn, Phys. Rev. B 35, 482 (1987).

[37] F. Offi, S. Iacobucci, P. Vilmercati, A. Rizzo, A. Goldoni, M. Sacchi, and G. Panaccione, Phys. Rev. B 77, 201101(R) (2008).

[38] S. Suga and A. Sekiyama, Photoelectron Spectroscopy: Bulk and Surface Electronic Structures, Springer Series in Optical Sciences Vol. 176 (Springer-Verlag, Berlin, 2013), pp. 266 and 270.

[39] At low temperature one can hardly remove the influence of the electrical charging effect caused by the photoelectron emission from the ELEPES spectra of the insulating iridates since the photoelectrons with very low kinetic energy are very sensitive to the effect.

[40] J. Kim, D. Casa, M. H. Upton, T. Gog, Y.-J. Kim, J. F. Mitchell, M. van Veenendaal, M. Daghofer, J. van den Brink, G. Khaliullin, and B. J. Kim, Phys. Rev. Lett. 108, 177003 (2012).

[41] K. Maiti, R. S. Singh, V. R. R. Medicherla, S. Rayaprol, and E. V. Sampathkumaran, Phys. Rev. Lett. 95, 016404 (2005).

[42] R. S. Singh, V. R. R. Medicherla, Kalobaran Maiti, and E. V. Sampathkumaran, Phys. Rev. B 77, 201102(R) (2008). 